Article

\title{
Functional Analysis of M-Locus Protein Kinase Revealed a Novel Regulatory Mechanism of Self-Incompatibility in Brassica napus L.
}

\author{
Fang Chen ${ }^{1,+}$, Yong Yang ${ }^{1,+}+\mathbb{D}$, Bing Li ${ }^{1}$, Zhiquan Liu ${ }^{1}$, Fawad Khan ${ }^{1}$, Tong Zhang ${ }^{2}$, \\ Guilong Zhou ${ }^{1}$, Jinxing Tu ${ }^{1} \mathbb{C}$, Jinxiong Shen ${ }^{1}$, Bin $\mathrm{Yi}^{1}{ }^{1}$, Tingdong $\mathrm{Fu}^{1}{ }^{1}$, Cheng Dai ${ }^{1, * \mathbb{C}}$ and \\ Chaozhi Ma ${ }^{1, *}$ \\ 1 National Key Laboratory of Crop Genetic Improvement, National Center of Rapeseed Improvement in \\ Wuhan, Huazhong Agricultural University, Wuhan 430070, China \\ 2 Key Laboratory of Horticultural Plant Biology, Ministry of Education, Huazhong Agricultural University, \\ Wuhan 430070, China \\ * Correspondence: cdai@mail.hzau.edu.cn (C.D.); yuanbeauty@mail.hzau.edu.cn (C.M.); \\ Tel.: +86-027-87281676 (C.D.); +86-027-87281676 (C.M.) \\ + These authors contributed equally to this work.
}

Received: 23 May 2019; Accepted: 4 July 2019; Published: 5 July 2019

check for updates

\begin{abstract}
Self-incompatibility (SI) is a widespread mechanism in angiosperms that prevents inbreeding by rejecting self-pollen. However, the regulation of the SI response in Brassica napus is not well understood. Here, we report that the M-locus protein kinase (MLPK) BnaMLPKs, the functional homolog of BrMLPKs in Brassica rapa, controls SI in B. napus. We identified four paralogue $M L P K$ genes in B. napus, including BnaA3.MLPK, BnaC3.MLPK, BnaA4.MLPK, and BnaC4.MLPK. Two transcripts of BnaA3.MLPK, BnaA3.MLPKf1 and BnaA3.MLPKf2, were generated by alternative splicing. Tissue expression pattern analysis demonstrated that BnaA3.MLPK, especially BnaA3.MLPKf2, is highly expressed in reproductive organs, particularly in stigmas. We subsequently created RNA-silencing lines and CRISPR/Cas9-induced quadruple mutants of BnaMLPKs in B. napus SI line S-70. Phenotypic analysis revealed that SI response is partially suppressed in RNA-silencing lines and is completely blocked in quadruple mutants. These results indicate the importance of BnaMLPKs in regulating the SI response of $B$. napus. We found that the expression of SI positive regulators S-locus receptor kinase $(S R K)$ and Arm-Repeat Containing 1 (ARC1) are suppressed in bnmlpk mutant, whereas the self-compatibility (SC) element Glyoxalase I (GLO1) maintained a high expression level. Overall, our findings reveal a new regulatory mechanism of $M L P K$ in the SI of B. napus.
\end{abstract}

Keywords: Brassica napus; self-incompatibility; self-compatibility; $M$ locus protein kinase (MLPK); RNAi; CRISPR/Cas9

\section{Introduction}

Self-incompatibility (SI) is an elaborate mechanism that promotes outcrossing and maintains genetic diversity in many flowering plants [1]. In the Brassicacease, SI is sporophytically regulated by a single genetic locus called the S-locus, at which multiple variants (now known as $S$ haplotype) occur in any one species [2]. The S-locus encodes the stigma determinant of SI, the S-locus receptor kinase gene $(S R K)$, which is a membrane-anchored Ser/Thr kinase localized in the plasma membrane of stigmatic papilla cells [3], and the pollen determinant of SI, a small secreted peptide localized in the pollen coat, which is known as S-locus cysteine-rich protein (SCR) [4] or the S-locus protein 11 (SP11) [5]. Biochemical studies have shown that SCR/SP11 is the ligand for SRK that are encoded in the same $S$ haplotype [6,7]. This $S$ haplotype-specific receptor-ligand interaction, and the resulting 
activation of a pollen-inhibitory signaling pathway upon self-pollination but not cross-pollination, explains the specificity of the SI response [8].

Some elements of SRK-mediated signaling have been reported in Brassica species. One report indicated that prior to self-incompatible pollination, SRK kinase activity is inhibited by the thioredoxin $h$ proteins THL1 and THL2 [9]. Once self-incompatible pollen lands on the stigma, the interaction of the SRK extracellular domain with its pollen ligand is thought to separate the THL1 and THL2 proteins from SRK, allowing the activation of SRK and its downstream signaling [9]. Subsequently, the plasma-membrane-tethered M-locus protein kinase (MLPK) is thought to interact with the activated SRK [10]. Another SRK interactor is the arm-repeat-containing protein ARC1, an E3 ubiquitin ligase [11], that can ubiquitinate Exo70A1 and direct this putative component of the exocyst for degradation by the proteasome [12]. Degradation of Exo70A1 is thought to inhibit the exocytosis of multivesicular bodies, presumably precluding the release of factors required for successful pollen tube growth (compatibility factors) and causing rejection of self-pollen [13]. ARC1 was reported to cause the degradation of at least one compatibility factor, glyoxalase I (GLO1) [14], a protein that is required for the detoxication of methylglyoxal, the cytotoxic by-product of glycolysis [15]. So far, the involvements of THL1/2, MLPK, ARC1, and Exo70A1 in SI have been reported only in a subset of Brassica species. However, their roles remain controversial [16,17] given studies in transgenic Arabidopsis thaliana self-incompatible SRK/SCR plants that found no evidence for the proposed roles of these genes in SI [16,18].

In this study, we examined the role of MLPK in the SI response of Brassica napus. MLPK was originally identified by positional cloning using $F_{2}$ populations derived from a cross between a self-incompatible Brassica rapa $\mathrm{S}_{8}$ homozygote $\left(S_{8} S_{8} M M\right)$ and a self-compatible B. rapa mutant Yellow Sarson C634 $\left(S_{f 2} S_{f 2} m m\right)$ [19]. The ability to reject self-pollen was restored in mutant papilla cells by transient expression of wild-type BrMLPK in these cells, and bimolecular fluorescence complementation (BiFC) experiments indicated that BrMLPK interacts with SRK in vitro [10]. These results suggest that $B r M L P K$ functions as a positive self-incompatibility factor in the SI signaling pathway of B. rapa. However, several questions regarding the role of $M L P K$ remain unresolved in SI. First, this role has not been confirmed in stably-transformed Yellow Sarson mutant plants. Second, functional analysis of MLPK has only been performed in B. rapa. Third, AtAPK1b, the A. thaliana gene that shares the highest sequence identity with $B r M L P K$, was reported to play no role in the SI response of self-incompatible transgenic $A$. thaliana [20]. Thus, the function of MLPK may vary among Brassicaceae species and how this protein might be involved in the SI response of the allotetraploid B. napus is still unknown.

To resolve these issues, we used a homology-based candidate gene approach to clone BnaMLPK sequences from a self-incompatible B. napus strain. Here, we report the identification and analysis of the four paralogues of BrMLPK in B. napus. We show that transgenic plants generated by RNA interference (RNAi)-based silencing and CRISPR/Cas9-based gene editing of BnaMLPKs exhibit partial and complete breakdown of SI, respectively. We show that this breakdown is associated with a drastic reduction of $S R K$ transcripts in mutant stigmas. Our results reveal a novel function of BnaMLPK as a positive regulator of the expression of SI-relevant genes in Brassicaceae stigmas.

\section{Results}

\subsection{Cloning and Sequence Analysis of BnaMLPKs from Self-Incompatibile B. napus}

To investigate the function of MLPK in self-incompatible B. napus, the genome sequence of MLPK was obtained from the Damor-bzh genome database [21] using BrMLPK as the reference sequence. B.napus possesses four MLPK genes: BnaA3.MLPK, BnaC3.MLPK, BnaA4.MLPK, and BnaC4.MLPK. Then, the $M L P K$ sequences from self-incompatible B. napus S-70 were cloned by PCR based on Damor-bzh MLPK sequences. The genome sequence of different paralogue genes ranged from 2080 to 2288 base pairs (bp), and the CDS sequence ranged from 1179 to $1306 \mathrm{bp}$ (Table S1). BnaA3.MLPK and BnaC3.MLPK contain six exons and five introns. BnaA4.MLPK and BnaC4.MLPK contain five exons and four introns. The sequence identification of the four MPLK proteins and nucleotides is very 
high (Table 1; Figure S1). BnaA3.MLPK, BnaC3.MLPK, BnaA4.MLPK, and BnaC4.MLPK are closer to BrMLPKf1/2, BoMLPKf1, BoMLPKn1, and BoMLPKn1, respectively (Table 2; Figure S1).

Table 1. Sequence analysis of CDS and proteins of MLPKs in Brassica napus.

\begin{tabular}{ccccc}
\hline Gene & BnaA3.MLPK & BnaC3.MLPK & BnaA4.MLPK & BnaC4.MLPK \\
\hline BnaA3.MLPK & - & $98.47 \%$ & $74.88 \%$ & $74.88 \%$ \\
BnaC3.MLPK & $98.56 \%$ & - & $75.60 \%$ & $75.60 \%$ \\
BnaA4.MLPK & $84.73 \%$ & $84.82 \%$ & - & $99.76 \%$ \\
BnaC4.MLPK & $84.82 \%$ & $84.90 \%$ & $98.09 \%$ & - \\
\hline
\end{tabular}

The percentages on below the diagonal line indicate CDS homology of MLPKs. The percentages on above the diagonal line indicate protein homology of MLPKs.

Table 2. Sequence alignment of the $M L P K$ transcripts in Brassica species.

\begin{tabular}{ccccccc}
\hline Transcripts & BrMLPKf1 & BrMLPKf & BrMLPKn & BoMLPKf1 & BoMLPKf2 & BoMLPKn \\
\hline BnaA3.MLPK & $99.83 \%$ & $99.32 \%$ & $81.34 \%$ & $98.64 \%$ & $93.29 \%$ & $84.82 \%$ \\
BnaC3.MLPK & $98.56 \%$ & $98.05 \%$ & $83.55 \%$ & $98.90 \%$ & $94.14 \%$ & $84.90 \%$ \\
BnaA4.MLPK & $85.93 \%$ & $84.02 \%$ & $91.77 \%$ & $86.01 \%$ & $80.03 \%$ & $98.09 \%$ \\
BnaC4.MLPK & $86.01 \%$ & $84.10 \%$ & $91.69 \%$ & $86.09 \%$ & $80.03 \%$ & $100.00 \%$ \\
\hline
\end{tabular}

\subsection{Sequence Analysis of BnaMLPK Transcripts}

Previous studies indicated that two isoforms of BrMLPK and BoMLPK are generated by alternative splicing $[10,22]$. We wondered if the alternative splicing still exists in BnaMLPKs. To confirm this hypothesis, we cloned the transcript sequences of BnaMLPK in S-70 using primers designed based on two isoforms of BrMLPK (BrMLPKf1 and BrMLPKf2) sequences (Table S2). Two different sequences were detected by Sanger sequencing (Table S1), which were transcribed by BnaA3.MLPK (Table S1; Figure 1A), named BnaA3.MLPKf1 and BnaA3.MLPKf2. BnaA3.MLPKf1 starts to transcribe from the first exon to the last exon, whereas the transcriptome of BnaA3.MLPKf2 initiates at the 475th base of the first intron (Figure 1A). The sequence length of BnaMLPKf2 transcript is $54 \mathrm{bp}$ longer than that of BnaMLPLf1, which was detected by PCR (Figure 1B). However, other MLPK paralogues contain one transcript. Therefore, a total of five transcripts were obtained from the four BnaMLPK genes. The domain analysis result indicated that, except for BnaA3.MLPKf2, the other four BnaMLPK proteins contain a typical plant $\mathrm{N}$-myristylation consensus sequence at the $\mathrm{N}$-terminal [23,24] (Figure 1C). The C-terminal of these five proteins is conserved, which contains a protein kinase domain (Figure 1D).
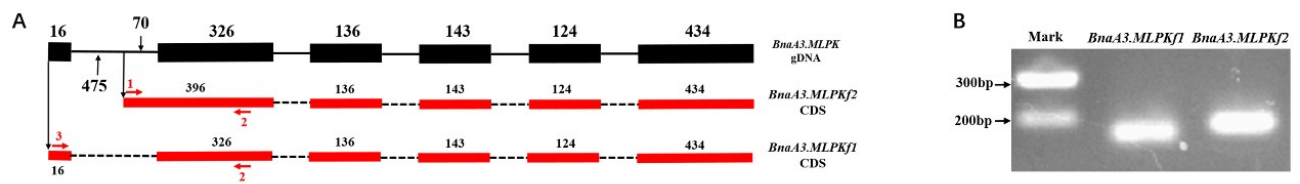

C
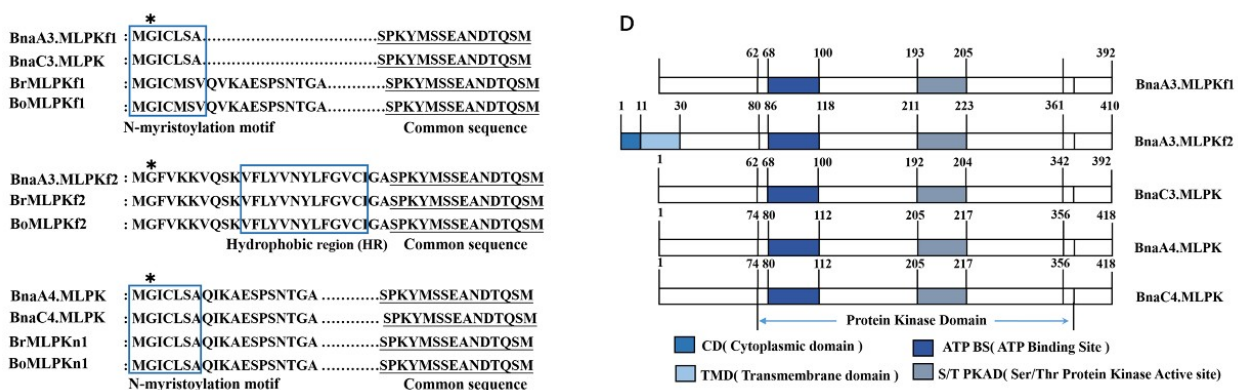

Figure 1. Comparison of the N-terminal amino acid sequences of MLPK in Brassica. (A) Schematic diagram of nucleotide sequences of the alternative transcription region of the BnaA3.MLPK gene. The black numbers indicate the base numbers. Red numbers and arrows mean the positions of primers used 
in (B). (B) The electrophoretogram of BnaA3.MLPKf1 and BnaA3.MLPKf2 transcripts. (C) Comparison of the deduced amino acid sequences of MLPK in Brassica. The blue boxes indicate the predicted myristylation consensus sequences in BnaA3.MLPKf1, BnaC3.MLPK, BrMLPKf1, BoMLPKf1, BnaC4.MLPKn1, BnaA4.MLPK, BrMLPKn1, and BoMLPKn1 and the specific hydrophobic regions in BnaA3.MLPKf2, BrMLPKf2, and BoMLPKf2. The common sequences are underlined. The conserved second-position Gly residues are indicated by asterisks. (D) Protein secondary structure prediction of BnaMLPKs.

\subsection{Phylogenetic and Tissue-Specific Expression Analysis of BnaMLPKS}

We explored the evolutionary relationship of MLPK proteins from various species using phylogenetic analysis. Four major clades, Clade A1, Clade A2, Clade B1, and Clade B2, were obtained (Figure 2A). BnaA3.MLPKf1 and BnaA3.MLPKf2 were in Clade B2, and BnaC3.MLPK was in Clade B1 (Figure 2A). BnaA4.MLPK and BnaC4.MLPK were in Clade A2 (Figure 2A). Then, quantitative real-time PCR (qRT-PCR) was performed to analyze the expression pattern of BnaMLPKs in different tissue. Both BnaA3.MLPK and BnaC3.MLPK are enriched in the stigma, but the expression of BnaA3.MLPK is much higher than BnaC3.MLPK (Figure 2B). The expressions of BnaA4.MLPK and BnaC4.MLPK are very low in all tissues (Figure 2B). Then, we tested different isoforms of BnaA3.MLPK in different tissues. The results indicate BnaA3.MLPKf2 is enriched in the stigma and petal, which is much higher than BnaA3.MLPKf1 (Figure 2C). These results demonstrate that BnaA3.MLPK and BnaC3.MLPK might participate in the regulation of SI responses, and BnaA3.MLPKf2 is the major isoform expressed in the stigma.

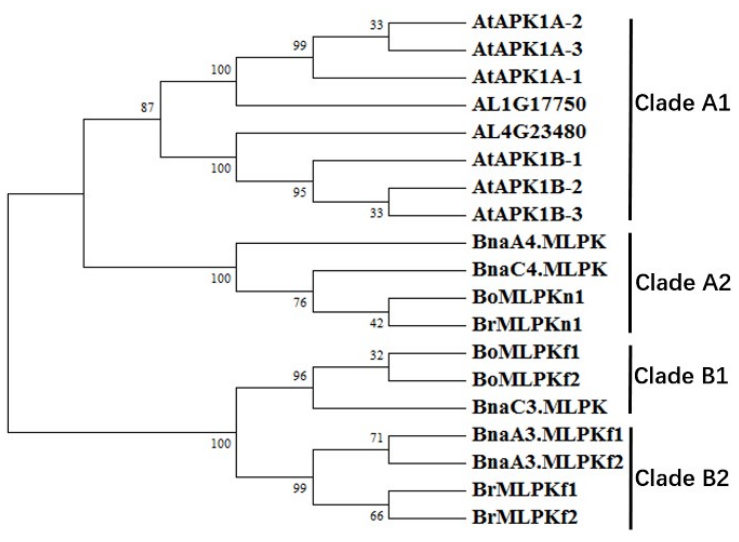

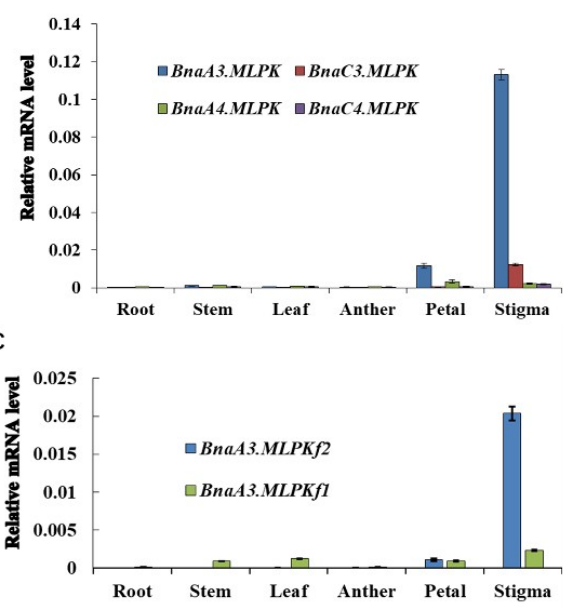

Figure 2. Phylogenetic and tissue-specific expression analysis of BnaMLPK isoforms. (A) The phylogenetic tree was analyzed using the neighbor-joining (NJ) algorithm using predicted MLPK amino acid sequences from B. napus, Brassica rapa, Brassica oleracea, Arabidopsis thaliana, and Arabidopsis lyrata. Confidence values from the bootstrap test (1000 replicates) are marked by the numbers on the tree. Clade A1, Clade A2, Clade B1, and Clade B2 indicate four sub-clades. BrMLPKf1, BrMLPKf2, and BrMLPKn1 are the proteins in B. rapa; AtAPK1A-1, AtAPK1A-2, AtAPK1A-3, AtAPK1B-1, AtAPK1B-2, and AtAPK1B-3 are the homologous proteins of BrMLPKs in A. thaliana; AL1G17750 and AL4G23480 are the homologous proteins of BrMLPKs in A. lyrata; BoMLPKf1, BoMLPKf2, and BoMLPKn1 are the homologous proteins of BrMLPKs in B. oleracea; and BnaA3.MLPKf1, BnaA3.MLPKf2, BnaC3.MLPK, BnaA4.MLPK, and BnaC4.MLPK are cloned homologous genes of BrMLPKs in B. napus. (B) Tissue-specific expression of the BnaA3.MLPK, BnaC3.MLPK, BnaA4.MLPK, and BnaC4.MLPK in different tissues of $B$. napus. The expression levels of each transcript were detected by real-time quantitative reverse transcription PCR (qPCR). The actin gene was used as control. (C) Tissue-specific expression of the two isoforms BnaA3.MLPKf1 and BnaA3.MLPKf2 in different tissues of B. napus. 


\subsection{RNAi Knockdown of BnaMLPKs Partially Suppressed SI Response in B. napus}

Though MLPK is a positive regulator of the SI response in B. rapa, the specific roles in B. napus remain unclear. To validate the function of BnaMLPKs in S-70 B. napus, we attempted to generate RNAi transgenic lines of BnaMLPKs using the stigma-specific SLR1 promoter to drive the expression of two hairpin RNA interference (hpRNAi) constructs [25], which were used to suppress the expression of BnaA3.MLPK/BnaC3.MLPK (RM1) and BnaA4.MLPK/BnaC4.MLPK (RM7) (Figure S2). The expressions of all four BnaMLPKs were partially suppressed in four RNAi transgenic lines, ranging from $25 \%$ to $60 \%$ (Figure 3A-D). Phenotypic analysis showed that the seed setting was partially rescued in bnmlpk-RNAi lines (Figure 3E), indicating that BnaMLPKs positively regulate SI response.
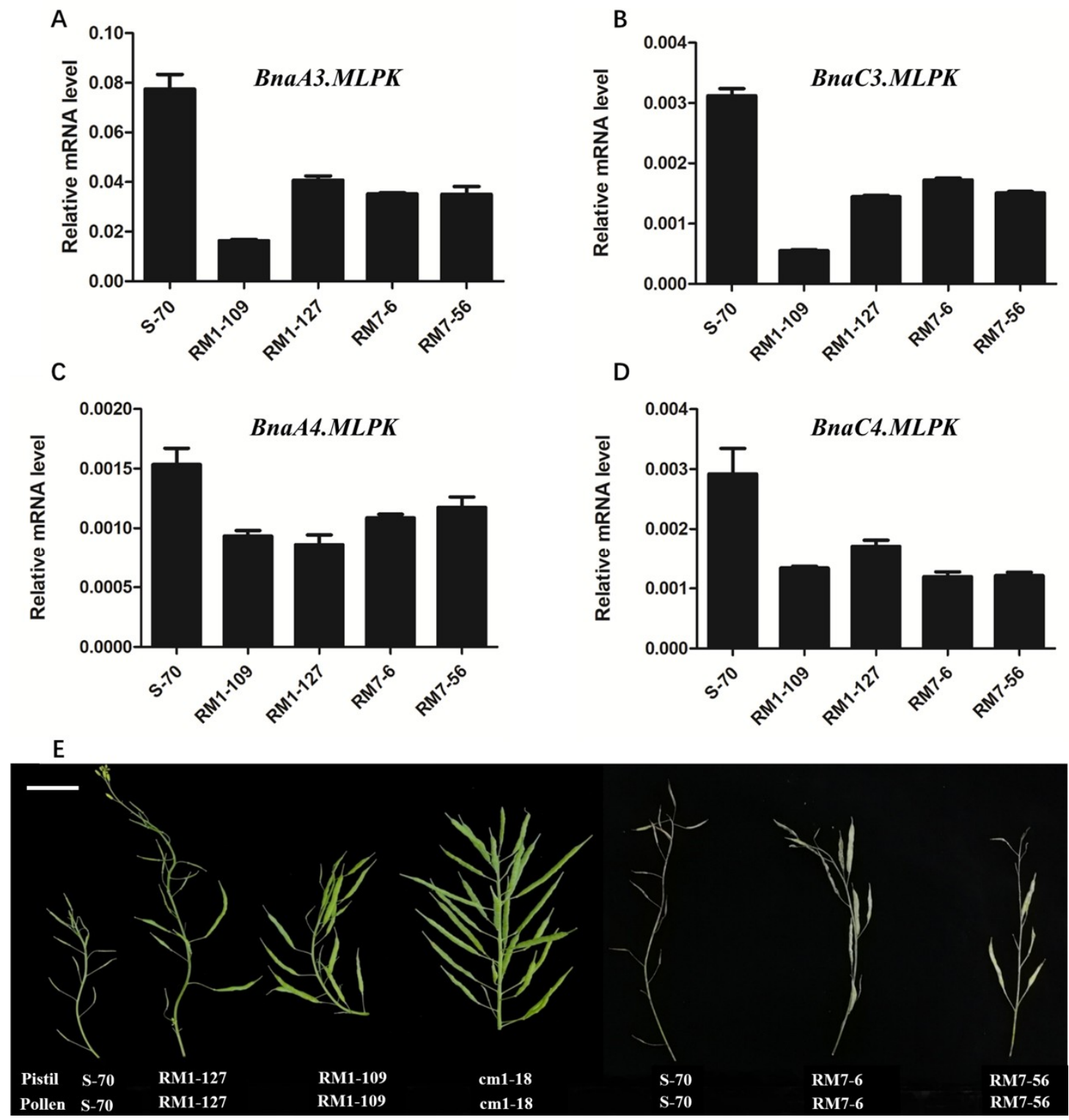

Figure 3. RNAi experiment demonstrated that $M L P K$ is required for incompatible pollination. (A-D) The expression analysis of BnaMLPKs in hpRNAi transgenic lines. The total RNA was extracted from at least thirty stigmas for each pollination treatment. Data represent the average of three technical replicates $( \pm \mathrm{SE})$. Similar expression results were acquired with three biological replicates. The actin gene was considered as the control reference. RM1-109 and RM1-127 indicate the two different positive 
plants from the RM1 hpRNAi construct. RM7-6 and RM7-56 denote the two transgenic plants from the RM7 hpRNAi construct. All four genes of MLPK were down-regulated in un-pollinated stigmas of RM1 and RM7 transgenic lines. The un-pollinated wild type S-70 was used as control. (E) Images of mature siliques from pistils of S-70, RM1-127, RM1-109, cm1-18, RM7-6, and RM7-56 lines following self-pollination. S-70 and cm1-18 were used as controls. Scale bar $=1 \mathrm{~cm}$.

\subsection{Knockout Mutant of BnaMLPKs Created Using CRISPR/Cas9 System Completely Breaks Down SI Response in B. napus}

The SI response was partially suppressed by knock down expression of BnaMLPKs in RNAi lines, but which paralogue is the dominant gene remained unclear. To address this question, two sgRNAs were designed to target the conserved sequences among the four paralogous genes (Figure S3A,B). By genetic transformation into S-70, we obtained six positive plants that were further confirmed by PCR using Cas9-specific primers (Figure S3C). Next, Sanger sequencing was used to assess the editing efficiency. The four BnaMLPK genes were examined in the six transgenic plants. As a result, the mutation frequency at the sgRNA1 target site ranged from $83.3 \%$ to $100 \%$ for each of the four genes, and at sgRNA2 target site, ranged from $66.7 \%$ to $100.0 \%$ (Table 3). Among all types of mutations, $16.7 \%(4 / 24)$ were deletions, $12.5 \%$ (3/24) were insertions, and $13.9 \%(17 / 24)$ were combined mutations (Figure S4).

Table 3. Percentages of mutated plants at the $\mathrm{T}_{0}$ generation by single-gene targeted sgRNAs.

\begin{tabular}{|c|c|c|c|c|c|c|}
\hline \multirow{2}{*}{ Target Gene } & \multirow{2}{*}{ sgRNA } & \multirow{2}{*}{$\begin{array}{l}\text { Number of Plants } \\
\text { Examined }\end{array}$} & \multirow{2}{*}{$\begin{array}{l}\text { Number of Plants } \\
\text { with Mutations }\end{array}$} & \multirow{2}{*}{$\begin{array}{c}\text { Mutation } \\
\text { Rate (\%) }\end{array}$} & \multicolumn{2}{|c|}{ Homozygous Mutations } \\
\hline & & & & & Number & Rate (\%) \\
\hline \multirow[t]{2}{*}{ BnaA3.MLPK } & sgRNA1 & 6 & 6 & 100.0 & 1 & 0.17 \\
\hline & sgRNA2 & & 6 & 100.0 & 0 & 0 \\
\hline \multirow{2}{*}{ BnaC3.MLPK } & sgRNA1 & 6 & 6 & 100.0 & 0 & 0 \\
\hline & sgRNA2 & & 6 & 100.0 & 0 & 0 \\
\hline \multirow[t]{2}{*}{ BnaA4.MLPK } & sgRNA1 & 6 & 5 & 83.3 & 0 & 0 \\
\hline & sgRNA2 & & 5 & 83.3 & 0 & 0 \\
\hline \multirow[t]{2}{*}{ BnaC4.MLPK } & sgRNA1 & 6 & 6 & 100.0 & 0 & 0 \\
\hline & sgRNA2 & & 4 & 66.7 & 0 & 0 \\
\hline
\end{tabular}

Both alleles for each gene might be mutated by CRISPR/Cas9, which could produce five genotypes: homozygote, bi-allele, heterozygote, chimera, and WT. To estimate the proportion of each genotype among the transgenic lines, we detected the mutation type of each targeted site using sequencing. All amplicons were analyzed by inserting them into a TA vector and sequencing 10 individual clones for each of the 48 amplicons. The genotype data are summarized in Table 4 . The genotyping results showed that $10.4 \%$ (5/48) sites were homozygous and $43.8 \%$ (21/48) sites were bi-allelic. Thus, a total of $54.2 \%$ sites had defects in both alleles (Table 4 ). The frequencies of heterozygotes and chimeras were $10.4 \%(5 / 48)$ and $25.0 \%(12 / 48)$, respectively. No mutations were found in $8.3 \%(4 / 48)$ of the sites.

Table 4. Genotypes of $\mathrm{T}_{0}$ transgenic plants.

\begin{tabular}{|c|c|c|c|c|c|c|c|}
\hline \multirow{2}{*}{ Target Gene } & \multirow{2}{*}{ Sites } & \multirow{2}{*}{$\begin{array}{c}\text { Number of } \\
\text { Examined Plants }\end{array}$} & \multicolumn{5}{|c|}{ Genotype } \\
\hline & & & Homozygote & Heterozygote & Bi-allele & Chimera & WT \\
\hline \multirow[t]{2}{*}{ BnaA3.MLPK } & sgRNA1 & 6 & $1(16.7 \%)$ & & $3(50.0 \%)$ & $2(33.3 \%)$ & \\
\hline & sgRNA2 & 6 & & & $3(50.0 \%)$ & $2(33.3 \%)$ & \\
\hline \multirow[t]{2}{*}{ BnaC3.MLPK } & sgRNA1 & 6 & & & $2(33.3 \%)$ & $4(66.7 \%)$ & \\
\hline & sgRNA2 & 6 & & & $4(66.7 \%)$ & $2(33.3 \%)$ & \\
\hline \multirow[t]{2}{*}{ BnaA4.MLPK } & sgRNA1 & 6 & $1(16.7 \%)$ & $1(16.7 \%)$ & $1(16.7 \%)$ & $2(33.3 \%)$ & $1(16.7 \%)$ \\
\hline & sgRNA2 & 6 & $1(16.7 \%)$ & $1(16.7 \%)$ & $3(50.0 \%)$ & & $1(16.7 \%)$ \\
\hline \multirow[t]{2}{*}{ BnaC4.MLPK } & sgRNA1 & 6 & $1(16.7 \%)$ & $2(16.7 \%)$ & $3(50.0 \%)$ & & \\
\hline & sgRNA2 & 6 & $1(16.7 \%)$ & $1(16.7 \%)$ & $2(33.3 \%)$ & & $2(33.3 \%)$ \\
\hline Total & & 48 & $5(10.4 \%)$ & $5(10.4 \%)$ & $21(43.8 \%)$ & $12(25.0 \%)$ & $4(8.3 \%)$ \\
\hline
\end{tabular}

Homozygote: the two alleles have the same mutation; Bi-allele: the two alleles have different mutations; Heterozygote: only one allele is mutated; Chimera: more than two different mutations exist; WT: wild type, no mutation. 
The six positive transgenic plants were self-crossed to obtain the $T_{1}$ population. Mutations of BnaMLPKs at the sgRNA1 and sgRNA2 target sites were examined in four plants $(\mathrm{cm} 1, \mathrm{~cm} 5, \mathrm{~cm} 7$, and $\mathrm{cm} 34$ ) from $\mathrm{T}_{1}$ lines. Among the transgenic plants, $\mathrm{cm} 1-18$ and $\mathrm{cm} 5-11$ lines were quadruple mutants of BnaMLPKs without wild-type sequence (Figure 4; Table 4). New mutation types were identified in the $\mathrm{T} 1$ generation of $\mathrm{cm} 5$. One possible reason is that Cas9 could be still functional at the non-mutated allele at the targeted region. Then, all the BnaMLPK genes showed down-regulated expression in the cm1-18 line compared with the wild-type S-70, especially BnaA3.MLPK, BnaA3.MLPKf2, BnaC3.MLPK, and BnaC4.MLPK (Figure 5A). Compared to the SI line S-70, abundant pollen germination, pollen tube elongation, and many seeds setting were observed in quadruple bnamlpk mutant after self-pollination (Figure 5B). When bnamlpk pollen was pollinated to S-70 stigmas, no pollen germination and seed setting were observed (Figure 5C), suggesting that BnaMLPKs did not change the pollen SI determination. These results demonstrate that BnaMLPK is a positive regulator of the SI response in B. napus.

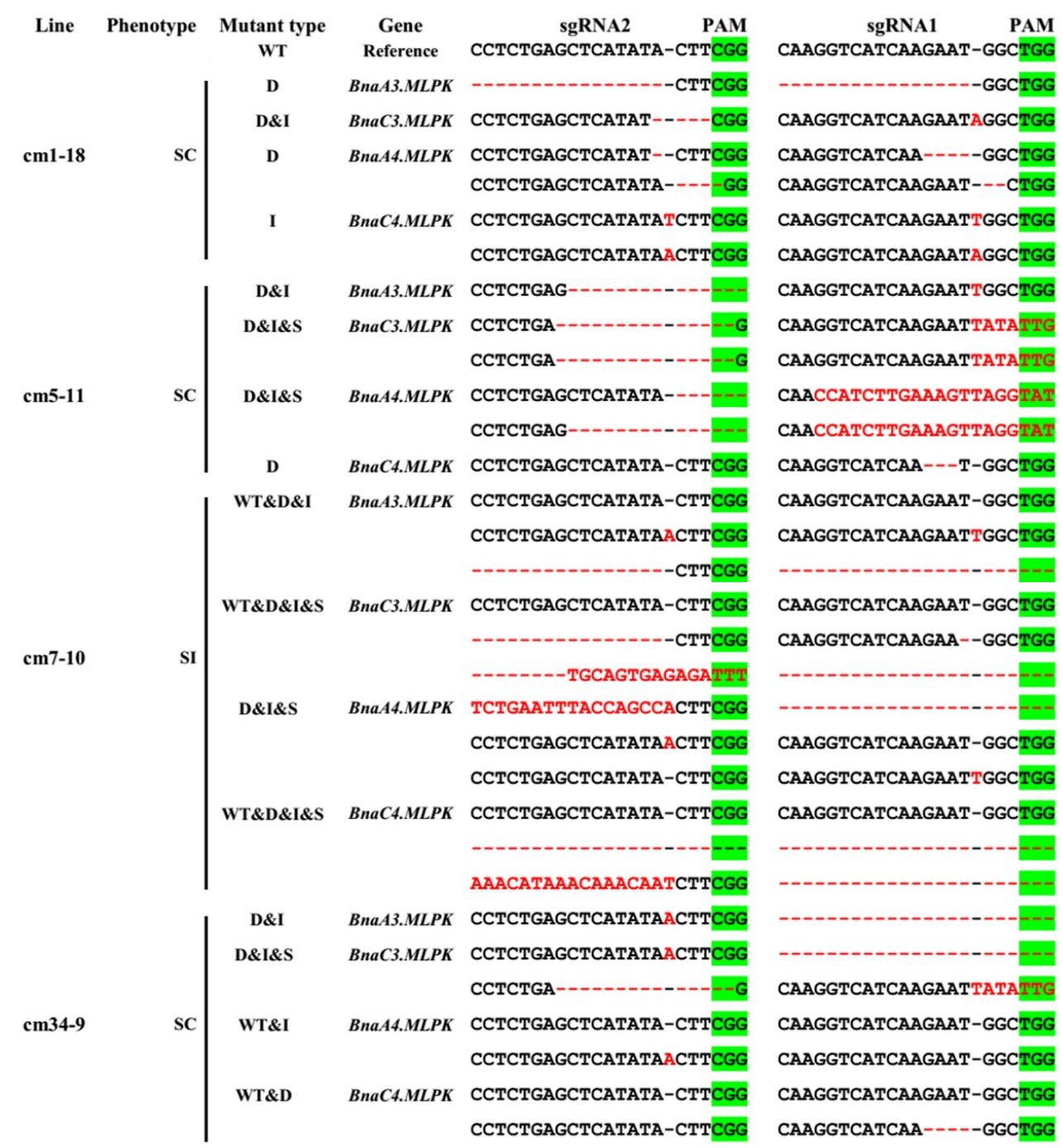

Figure 4. The editing information of BnaMLPKs in $\mathrm{T}_{1}$ generation mutants and sequencing analysis of the four $\mathrm{T}_{1}$ generation lines (named $\mathrm{cm} 1-18, \mathrm{~cm} 5-11, \mathrm{~cm} 7-10$, and $\mathrm{cm} 34-9$ ). The DNA was extracted from the leaves. Representative sequences of the mutated $M L P K$ were aligned with those of the reference gene. The protospacer adjacent motif (PAM) region is marked by green. The two sgRNAs are sgRNA1 and sgRNA2. The inconsistent region indicates that the sequence is edited, which is denoted by red. SI: self-incompatibility; SC: self-compatibility; D: deletion; I: insertion; S: substitution; WT: wild-type S-70 sequence. 

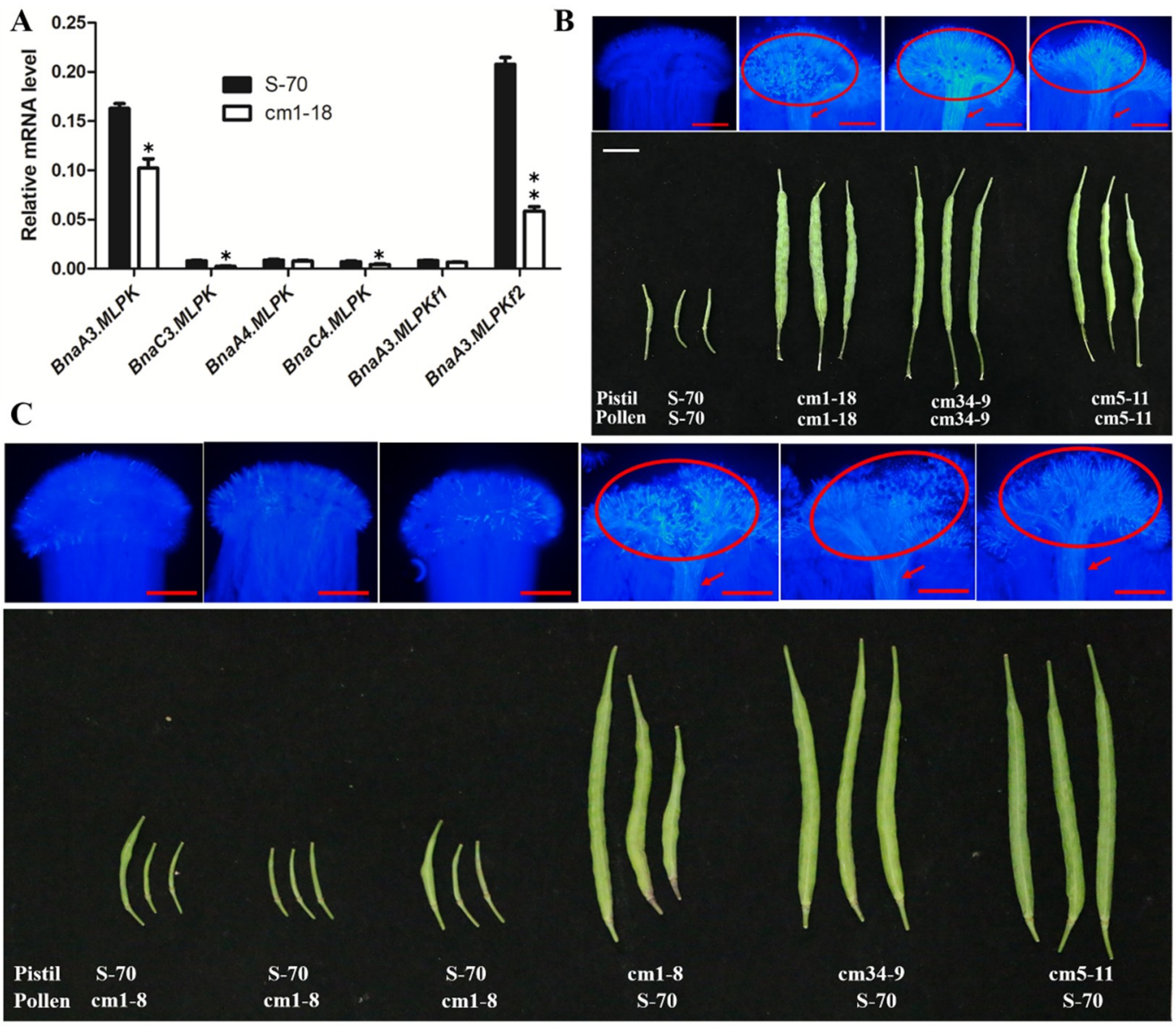

Figure 5. $M L P K$ is required for incompatible pollination in B. napus (A) Expression analysis of BnaMLPKs in bnamlpk mutant. The total RNA was extracted collectively from at least 30 stigmas for each pollination treatment. Data represent the average of three technical replicates $( \pm S E)$. Similar expression results were acquired with three biological replicates. Actin gene was used as the control reference. All four genes of MLPK were down-regulated in un-pollinated stigmas of bnamlpk mutants. The un-pollinated wild-type S-70 was used as the control. The asterisks represent significant differences (ANOVA: ${ }^{*} p<0.05,{ }^{* *} p<0.01$ ). The black bar indicates the stigmas of un-pollinated wild-type S-70. The white bar represents the stigmas of un-pollinated mutant $\mathrm{cm} 1-18$. (B) The upper plate indicates aniline blue staining. The aniline blue assays were performed $16 \mathrm{~h}$ after self-pollination. Red arrows indicate the pollen tubes. The red circles indicate germinated pollen grains. Scale bar $=50 \mu \mathrm{m}$. The lower plate represents mature siliques developed from pistils of S-70, cm1-18, cm34-9, and cm5-11 lines following pollination as indicated. Scale bar $=1 \mathrm{~cm}$. (C) The upper plate indicates aniline blue staining. The aniline blue assays were performed $16 \mathrm{~h}$ after different pollination treatments as indicated in the lower plate. Red arrows show the pollen tubes. The red circles indicate germinated pollen grains. Scale bar $=75 \mu \mathrm{m}$. The lower plate represents mature siliques developed from pistils of S-70, cm1-18, cm34-9, and cm5-11 lines following pollination as indicated. Scale bar $=1.5 \mathrm{~cm}$.

\subsection{Expression of SI-Related Genes Changed in bnamlpk Mutant}

Previous studies reported that SRK, ARC1, THL1/2, Exo70A1 and GLO1 genes are mainly involved in the SI of B. napus $[3,9,11,12,14,26]$. So, we wanted to know whether the SC phenotype of bnamlpk is caused by changing the expression of SI-related genes. qRT-PCR was used to detect the relative expression levels of these genes in the stigmas of bnamlpk mutant and wild type (S-70) after un-pollination (up) and self-pollination (sp). Without pollination, $S R K$ and $A R C 1$ were suppressed in bnamlpk compared to the S-70 (Figure 6A,B). After self-pollination, these two genes were down-regulated (SRK: 1.4 fold, ARC1: $\sim 1.2$ fold) in S-70, but not in bnamlpk (Figure 6A,B). The expression of GLO1 significantly decreased 
( 2.3 fold) in S-70 after self-pollination (Figure 6C). GLO1 was slightly up-regulated ( 1.3 fold) in bnamlpk without self-pollination (Figure 6C). The expression of the other genes showed almost no differences between the mutant and wild-type with or without self-pollination (Figure 6D-F). These results suggest that BnaMLPKs might regulate the expression of $S R K, A R C 1$, and GLO1 to control the SI response in B. napus.
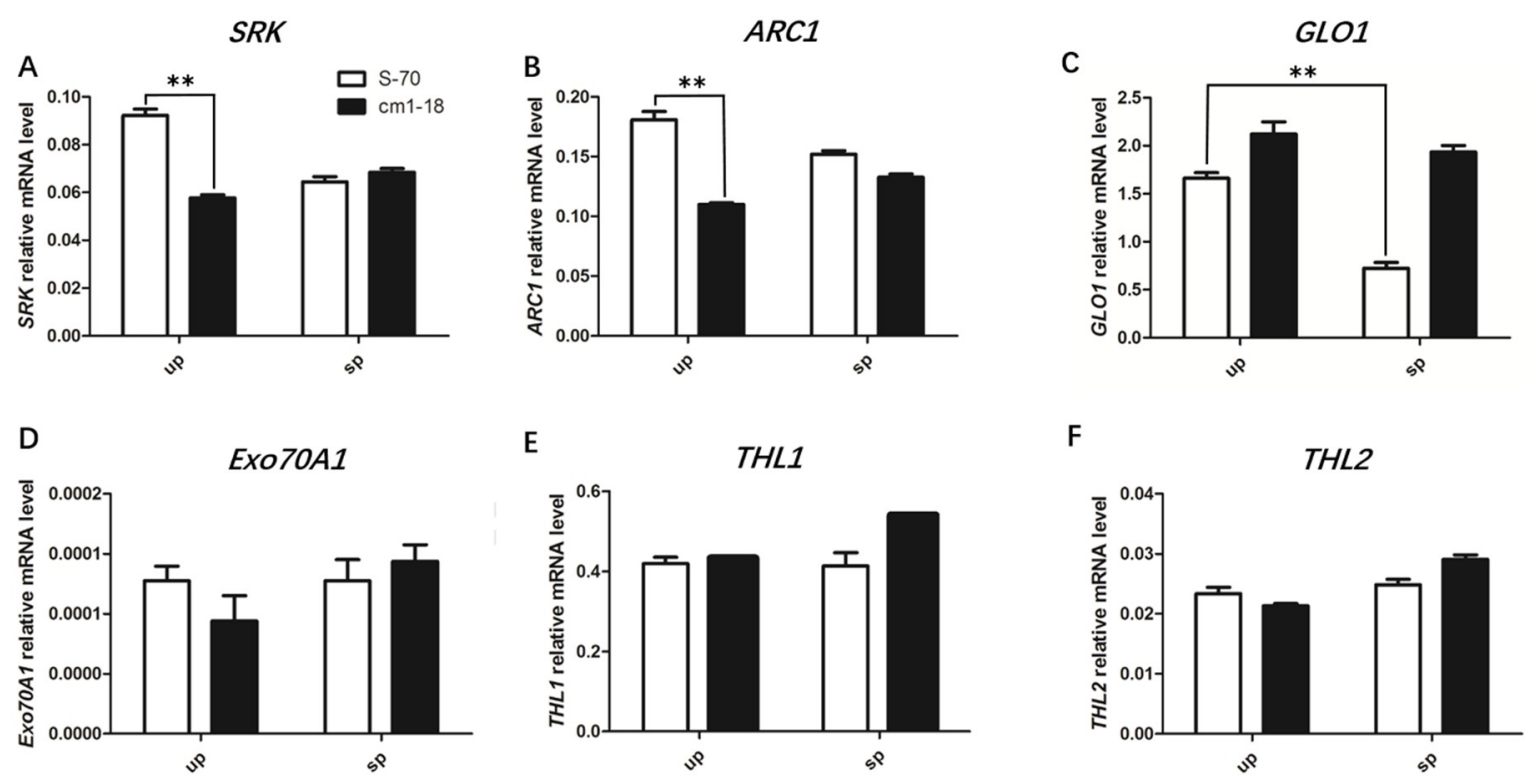

Figure 6. Quantitative real-time PCR analysis of the expression pattern of (A) $S R K,(\mathbf{B}) A R C 1,(\mathbf{C})$ GLO1, (D) Exo70A1, (E) THL1, and (F) THL2 genes in the stigmas of wild-type and mutant B. napus S-70. The total RNA was extracted collectively from at least 30 stigmas for each treatment. Data represent the average of three technical replicates $( \pm \mathrm{SE})$. Similar expression results were acquired with three biological replicates. The actin gene was used as the control reference. Asterisks indicate significant differences (ANOVA: ${ }^{* *} p<0.01$ ). The white bar indicates wild-type S-70 stigmas of un-pollination (up) or self-pollination (sp) and the black bar denotes the mutant cm1-18 stigmas of un-pollination (up) or self-pollination (sp).

\section{Discussion}

\subsection{BnaMLPK is a Positive Regulator of SI Response in B. napus}

SRK-mediated signaling has been reported in Brassica species [13,27-29]. MLPK, a plasma membrane-tethered M-locus protein kinase, is phosphorylated by SRK and required for the SI signaling pathway in B. rapa $[10,19]$. However, the homolog of BrMLPK in Arabidopsis plays no role in the SI response in self-incompatible transgenic $A$. thaliana (SRKb-SCRb) [20]. Therefore, more evidence is required to clarify the roles of $M L P K$ in the SI response of Brassicacea. In this study, knock-down and knock-out of BnaMLPKs was performed in self-compatible B. napus S-70, which broke down the SI response, suggesting that $B n a M L P K s$ are positive regulators of the SI response.

The sgRNA that targeted the conserved sequences among the four paralogous genes in the CRISPR/Cas9 system could in theory generate single, double, triple, and quadruple mutants. However, no single mutant bnamlpk line was separated in the present transgenic materials, probably due to the high genome editing efficiency. The $\mathrm{cm} 34-9$ line showed a SC phenotype (Figure 4), the genotypes of BnaA3.MLPK and BnaC3.MLPK were homozygous mutations, and BnaA4.MLPK and BnaC4.MLPK were heterozygous mutations. Based on the expression pattern and phenotype of the mutant, we propose that BnaA3.MLPK and BnaC3.MLPK are the functional genes of SI in B. napus. However, which paralogue gene plays the dominant function in the SI response remains unknown. The single mutants 
of BnaA3.MLPK and BnaC3.MLPK could be isolated in the next generation, which would help us to answer this question.

\subsection{Alternative Splicing Involved in MLPK-Regulated SI in B. napus}

Alternative splicing (AS) of eukaryotic transcripts is a mechanism that enables cells to generate vast protein diversities from a limited number of genes. Studies in several plants have indicated that tissue-specific AS mediates tissue differentiation and promotes specialized characteristics [30]. Alternative splicing at first exons (AFEs) has been well studied in mammals, which is suggested to contribute to the diversification of gene expression [31]. Transcript analysis showed that two isoforms of BnaA3.MLPK are generated by AFEs. The expression patterns of these two isoforms of BnaA3.MLPK are significantly different, indicating the regulation of AFEs in gene expression.

B. napus (AACC, $2 n=38$ ), as an allotetraploid species, was developed by natural allopolyploidization between B. rapa $(\mathrm{AA}, 2 n=20)$ and B. oleracea $(\mathrm{CC}, 2 n=18)$ [21]. MLPK plays an important role in the SI response of B. rapa [10], but not in transgenic A. thaliana (SRKb-SCRb) [10]. These results imply that the function of MLPK has been differentiated between Arabidopsis and Brassica during evolution [32]. BrMLPK was found to generate two transcripts, BrMLPLf1 and BrMLPKf2, by AFEs, both of which function in SI signal transduction in the stigma of $B$. rapa [10]. This study also demonstrates that BnaA3.MLPKf1 and BnaA3.MLPKf2 are the major isoforms of BnaA3.MLPK in B. napus. Further experiments are need to determine the functions of the two transcripts, such as a complementary experiment transforming two BnaA3.MLPK transcripts into the bnamlpk mutant by rescuing the SI phenotype.

\subsection{BnaMLPKs Regulate SI Responses by Influencing the Expression of SCR-SRK Pathway Components}

Several genes have been identified in the SRK-SCR pathway [33]. Although the regulatory mechanism of these genes is well-studied at the protein level, little has been reported about their regulatory mechanism at the transcription levels. We found the expressions of SRK and ARC1 were suppressed in bnamlpk before self-pollination (Figure 6A,B). Down-regulated expressions of $S R K$ and $A R C 1$ prevented the SI signal transduction, resulting in pollen tubes growth and elongation. Without pollination, the self-compatible factor GLO1 was slightly induced in bnamlpk. These results imply that the inhibition of $S R K$ and $A R C 1$ and the maintained high level of GLO1 cause the breaking down of the SI response in bnamlpk. The mechanism through which MLPK regulates the expressions of SRK, ARC1, and GLO1 needs to be further investigated.

Given the similar sequences, expression patterns, and transcriptional processing of BnaMLPK and its $B$. rapa homologue $B r M L P K$, the expectation is that the BnaMLPK protein would be required for the SI response of $B$. napus and would have the features and function previously reported for the BrMLPK protein, i.e., plasma-membrane localization, interaction with the SRK receptor, and involvement in SRK-mediated signaling. Consistent with previous reports, we found that down-regulation or disruption of BnaMLPK caused partial or complete loss of SI, thus confirming a role of BnaMLPK in SI. However, our results show that the self-compatibility phenotype of bnmlpk mutants is associated with a drastic reduction in the level of $S R K$ transcripts in un-pollinated stigmas, a reduction that is known to cause breakdown of SI [34,35]. Although we did not investigate the signaling role of the BnaMLPK protein, we did reveal a novel role of BnaMLPK that had not been reported for BrMLPK. BnaMLPK may act independently of SRK signaling to regulate the transcript levels of genes required for SI. Further analysis is required to understand how this SRK-independent signaling regulatory function is achieved and if it involves transcriptional control in the nucleus by as-yet-unknown transcription factors or post-transcriptional control of transcript stability in the cytoplasm. 


\section{Materials and Methods}

\subsection{Plant Materials and Growth Condition}

The self-incompatible B. napus S-70 species was separated from the self-incompatible S-1300, which was acquired by interspecific hybridization between the self-compatible $B$. napus line Huayou8 and the self-incompatible B. rapa Xishuibai [36]. S-70 and its relative transgenic lines were grown in the transgenic field of Huazhong Agriculture University, Wuhan, China, during B. napus growing season, or cultivated in a growth room under the light intensity of $100 \mu \mathrm{mol} \cdot \mathrm{m}^{-2} \mathrm{~s}^{-1}$ with a $16 / 8 \mathrm{~h}$ light/dark photoperiod at $22^{\circ} \mathrm{C}$.

\subsection{Sequence Cloning of BnaMLPKs}

To clone the genome DNA (gDNA) or CDS region of MLPKs, the B. napus genomic DNA or total RNA was extracted from leaves or stigmas following the CTAB method [37] or plant mini RNeasy kit (Qiagen, Hilden, Germany) according to the manufacturer's protocol. Approximately $1 \mu \mathrm{g}$ of total RNA was used for cDNA synthesis with the Thermo Scientific Revertaid First Strand cDNA Synthesis kit (Thermo, New York, USA). Primers M4CDS-F/M4CDS-R were used to amplify the four paralogues of the MLPK genes (Table S2), followed by standard PCR procedure: $98^{\circ} \mathrm{C}$ for $2 \mathrm{~min}$; $98^{\circ} \mathrm{C}$ for $20 \mathrm{~s}$, $55^{\circ} \mathrm{C}$ for $20 \mathrm{~s}, 72{ }^{\circ} \mathrm{C}$ for $1.5 \mathrm{~min}, 35 \mathrm{cycles} ; 7{ }^{\circ} \mathrm{C}$ for $10 \mathrm{~min}$; and $25^{\circ} \mathrm{C}$ for $5 \mathrm{~min}$. Two primer pairs (Table S2), BrMLPKf1-F/BrMLPKf1-R and BrMLPKf2-F/BrMLPKf2-R, were designed according to the BrMLPKf1/2 cDNA sequence [10,22], which were used to amplify the transcripts of BnaMLPK. PCR was performed using the following procedure: $98{ }^{\circ} \mathrm{C}$ for $2 \mathrm{~min} ; 98^{\circ} \mathrm{C}$ for $20 \mathrm{~s}, 55^{\circ} \mathrm{C}$ for $20 \mathrm{~s}, 72{ }^{\circ} \mathrm{C}$ for $1 \mathrm{~min}, 35$ cycles; $72{ }^{\circ} \mathrm{C}$ for $10 \mathrm{~min}$; and $25^{\circ} \mathrm{C}$ for $5 \mathrm{~min}$. All PCR products were purified and ligated into the pMD18-T vector (Takara, Wuhan, China). Subsequently, the positive clones were confirmed via sequencing (TsingKe, Wuhan, China).

\subsection{Tissue-Specific Expression Analysis of BanMLPKs}

Total RNA from different tissues was extracted using a Plant Total RNA Isolation Kit (Sangon Biotech, Shanghai, China, No. SK8631) following the manufacturer's instructions. Approximately $1 \mu \mathrm{g}$ of total RNA was used for cDNA synthesis using a PrimeScriptTM RT reagent kit (TaKaRa, Tokyo, Japan, Cat\#RR047A). A $10 \mu \mathrm{L}$ total volume of reaction mixture was used for qPCR, which contained $5 \mu \mathrm{L} 2 \times$ SYBR Green master mix (Achard et al., 172-5124, BioRad), $0.5 \mu \mathrm{L} 5 \times$ diluted cDNA, $0.25 \mu \mathrm{L}$ of

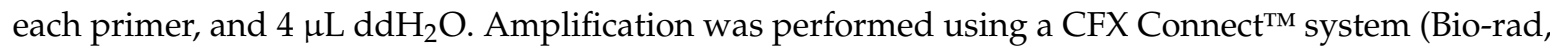
USA). The amplification program involved one cycle of $95^{\circ} \mathrm{C}$ for $5 \mathrm{~min}$, followed by 50 cycles of $95^{\circ} \mathrm{C}$ for $15 \mathrm{~s}, 60{ }^{\circ} \mathrm{C}$ for $20 \mathrm{~s}$, and $72{ }^{\circ} \mathrm{C}$ for $20 \mathrm{~s}$. The fluorescent products were detected at the third step of each cycle. The expression level of each gene was calculated using the $2^{-\Delta \Delta C T}$ method. All analyses were repeated with three biological replicates. The actin gene (Gene-Bank accession no: AF111812) served as the internal control. All primers are listed in Table S2.

\subsection{Phylogenetic Analysis}

The genomic, full coding DNA sequence (CDS), and protein sequences of four paralogue Brassica napus MLPKs were aligned by DNAMAN (version5.2.2, Lynnon BioSoft, USA, https://www.lynnon. $\mathrm{com} / \mathrm{pc} /$ framepc.html).

For phylogenetic analysis, homolog sequences MLPK in A. thaliana and A. lyrata were obtained from the TAIR Web site (https://www.arabidopsis.org/). The BrMLPK and BoMLPK protein sequences were used as previously reported [22]. The BnaMLPK protein sequences were obtained from DNAMAN-predicted CDS sequence. The sequence alignment was performed using Clustal Omega (http://www.ebi.ac.uk/Tools/msa/clustalo). An unrooted phylogenetic tree was constructed using MEGA7 (http://www.megasoftware.net), and aligned with default parameters (gap opening penalty $=10$, gap extension penalty $=0.1)$, the neighbor-joining $(\mathrm{NJ})$ statistical method, bootstrap analysis (1000 replicates), and pairwise gap deletion mode. 


\subsection{Plasmid Construction and B. napus Transformation}

The GENE-sgRNA plant expression vectors were constructed following a previously reported method with minor modifications [38]. The target sgRNA sequences were designed using the web server CRISPR-P (http://cbi.hzau.edu.cn/cgi-bin/CRISPR;). Using $p C B C-D T 1 T 2$ as the template, two AtU6 promoter-sgRNA-AtU6 terminator cassettes were amplified by PCR using the primers listed in Table S2. The PCR fragments were inserted into pKSE401 by Golden Gate Assembly [39], and confirmed by Sanger sequencing. These vectors were then used for plant transformation.

The hairpin RNA interference (hpRNAi) construct was reformed from the pCAMBIA2300 vector. The reformed construct contains a stigma-specific SLR1 promoter [40], an intron, and a fragment of poly A (Figure S2). The sense fragment with nucleotides 908 to 1107 from the coding sequence of BnaA3.MLPK ( $\left.\mathrm{CDS}_{908-1107}\right)$, including KpnI at $\mathrm{N}$-terminal and SacI at the C-terminal, was amplified and inserted between the intron and the poly A. Then, the reverse complement fragment of CDS ${ }_{908-1107}$ with PstI at the $\mathrm{N}$-terminal and $\mathrm{XbaI}$ at the $\mathrm{C}$-terminal was developed and inserted between the SLR1 promoter and the intron. Similarly, the sense fragment with nucleotides 1007 to 1206 from the coding sequence of BnaC4.MLPK ( $\left.\mathrm{CDS}_{1007-1206}\right)$, including KpnI at the N-terminal and SacI at theC-terminal, was amplified and inserted between the intron and the poly $\mathrm{A}$. Then, the reverse complement fragment of $\mathrm{CDS}_{1007-1206}$ with PstI at the N-terminal and XbaI at the C-terminal was developed and inserted between the SLR1 promoter and the intron. The reconstructed vector was transformed into Agrobacterium GV3103 for plant transformation.

The Agrobacterium-mediated transformation was completed [41]. The explants were incubated in the Agrobacterium-infection buffer (MS, $4.43 \mathrm{~g} \cdot \mathrm{L}^{-1}$; sucrose, $30 \mathrm{~g} \cdot \mathrm{L}^{-1}$; acetosyringone, $100 \mathrm{mM} \cdot \mathrm{L}^{-1}$; $\mathrm{pH}$ 5.8-5.9) for $20 \mathrm{~min}$, and then transferred to M1 medium plates (MS, $4.43 \mathrm{~g} \cdot \mathrm{L}^{-1}$; sucrose, $30 \mathrm{~g} \cdot \mathrm{L}^{-1}$; acetosyringone, $100 \mathrm{mM} \cdot \mathrm{L}^{-1}$; mannitol, $18 \mathrm{~g} \cdot \mathrm{L}^{-1}$; 2,4-D, $1 \mathrm{mg} \cdot \mathrm{L}^{-1}$; kinetin, $0.3 \mathrm{mg} \cdot \mathrm{mL}^{-1}$; $\mathrm{pH}, 5.8-5.9$ ). The explants were kept in dark for $48 \mathrm{~h}$. Afterward, the explants were transferred to M2 medium plates (MS, $4.43 \mathrm{~g} \cdot \mathrm{L}^{-1}$; sucrose, $30 \mathrm{~g} \cdot \mathrm{L}^{-1}$; acetosyringone, $100 \mathrm{mM} \cdot \mathrm{L}^{-1}$; mannitol, $18 \mathrm{~g} \cdot \mathrm{L}^{-1}$; $\mathrm{AgNO}_{3}, 4 \mathrm{mg} \cdot \mathrm{L}^{-1}$; 2,4-D, $1 \mathrm{mg} \cdot \mathrm{L}^{-1}$; kinetin, $0.3 \mathrm{mg} \cdot \mathrm{mL}^{-1}$; Timentin, $\left.270 \mathrm{mg} \cdot \mathrm{L}^{-1} ; \mathrm{pH}, 5.8-5.9\right)$ with appropriate antibiotics to induce callus growth. The calluses were transferred to M3 (MS, $4.43 \mathrm{~g} \cdot \mathrm{L}^{-1}$; glucose, $10 \mathrm{~g} \cdot \mathrm{L}^{-1}$; xylose, $0.25 \mathrm{~g} \cdot \mathrm{L}^{-1}$; zeatin, $2 \mathrm{mg} \cdot \mathrm{L}^{-1}$; IAA, $0.1 \mathrm{mg} \mathrm{L}{ }^{-1}$; Timentin, $\left.270 \mathrm{mg} \cdot \mathrm{L}^{-1} ; \mathrm{pH}, 5.8-5.9\right)$, followed by culturing in M4 medium (MS, $2.22 \mathrm{~g} \cdot \mathrm{L}^{-1}$; sucrose, $10 \mathrm{~g} \cdot \mathrm{L}^{-1}$; IBA, $0.5 \mathrm{mg} \mathrm{L}^{-1}$; Timentin, $135 \mathrm{mg} \cdot \mathrm{L}^{-1}$; $\mathrm{pH}, 5.8-5.9$ ) to allow the regeneration of shoots and roots.

\subsection{Mutant Screening and Validation of Genome Editing}

To analyze the mutations caused by CRISPR/Cas9, genomic DNA was extracted from leaves using the CTAB method [37]. The flanking sequences of the CRISPR target sites were amplified by PCR using gene-specific primers (Table S2). Then, most of the amplicons were directly sequenced. To decode mutations, the online tool DSDecode (http://dsdecode.scgene.com/;) was used for chromatogram decoding. The sequences files and the reference gene sequences were uploaded to the server and analyzed using default settings. The results were aligned with the reference sequences to ensure that the mutations were in the sgRNA targeted sites. For complex mutations, the amplicons were first sub-cloned into the $p$ GEM18-T vector (Cat\#A3610, Takara, Tokyo, Japan), and about 10 clones for each amplicon were individually sequenced. To analyze the RNAi lines, we used specific primers to detect the positive lines including SLR1-RNAi function fragment (Table S2). All the transgenic plants, including CRISPR/Cas9 mutants, were transferred to soil for further analysis.

\subsection{Aniline Blue Staining Assay}

The aniline blue staining was performed as reported with minor modifications [42]. After $16 \mathrm{~h}$ of pollination, the pistils were collected from the flowers, and immediately fixed in 3:1 ethanol/glacial acetic acid for $2 \mathrm{~h}$. The samples were further softened with $1 \mathrm{M} \mathrm{NaOH}$ at $60^{\circ} \mathrm{C}$ for $1.5 \mathrm{~h}$ and then washed three times with distilled water. Pollen tubes were then stained by $0.1 \%$ decolorized aniline 
blue ( $\mathrm{pH} 9-11$, in $0.1 \mathrm{M} \mathrm{K}_{3} \mathrm{PO}_{4}$ ) and placed in the dark for $6 \mathrm{~h}$. The stained samples were observed under a fluorescence microscope (Ax10, Zeiss, Berlin, Germany) equipped with a UV filter set.

\subsection{Self-Incompatibility Assay}

Self-incompatibility phenotypic observation was performed [36]. At the flowering stage, the major inflorescence and two or three secondary ramifications were bagged for self-pollination after removing all blooming flowers artificially. The bags were shaken gently every two days to ensure sufficient self-pollination. The transgenic or wild-type flowers were cross pollinated after emasculation one day before anthesis. After two weeks, the bags were removed to allow the growth of seeds. The phenotypes were observed after maturation of seeds.

\section{Conclusions}

In this study, we cloned the BrMLPK homologous gene in B. napus, analyzed the transcripts in stigmas, and revealed their expression patterns in different tissues. We also demonstrated that BnaMLPKs positively regulate the SI response in the B. napus. Combined with the results from previous SI studies, we speculate that MLPK mediates the SI signaling pathway through regulating the expression of $S R K, A R C 1$, and GLO1. These findings not only strengthen our understanding of the molecular roles of MLPK in the SI response of B. napus, but also directly contribute to the future study of the molecular mechanisms of SI in Brassica.

Supplementary Materials: The following are available online at http://www.mdpi.com/1422-0067/20/13/3303/s1, Figure S1. RNA-silencing constructs used in this study; Figure S2. Information of CRISPR/Cas9 construct of editing BnMLPK; Figure S3. The editing information of BnMLPK in $\mathrm{T}_{0}$ generation mutants; Figure S4. The editing information of BnaMLPKs in T0 generation mutants; Table S1. The gDNA and CDS sequence of BnMLPK; Table S2. Primers used in the study.

Author Contributions: F.C. and Y.Y. designed and performed the research, analyzed data, and wrote the article with contributions from all the authors; F.C. and Y.Y. performed research and analyzed data; B.L., Z.L., T.Z., G.Z. and F.K. provided technical assistance to F.C. and Y.Y.; C.M., B.Y., J.S., J.T. and T.F. supervised the experiments; C.M. and C.D. supervised and complemented the writing.

Funding: This work was funded by the National Key Research and Development Program of China (2016YFD0100803), and partly by the National Science Foundation of China (31571706).

Acknowledgments: We thank anonymous reviewers for providing constructive comments. We thank Zuoxiong Liu from the Foreign Language School of HZAU for proof-reading and editing the English language of the manuscript.

Conflicts of Interest: All the authors declare that the research was conducted in the absence of any commercial or financial relationships that could be construed as a potential conflict of interest.

\section{References}

1. Nettancourt, D.D. Incompatibility and Incongruity in Wild and Cultivated Plants, 2nd ed.; Springer: New York, NY, USA, 2001; Volume 3, p. 347.

2. Bateman, A.J. Self-incompatibility systems in angiosperms. III. Cruciferae. Heredity 1955, 9, 52-68. [CrossRef]

3. Stein, J.C.; Howlett, B.; Boyes, D.C.; Nasrallah, M.E.; Nasrallah, J.B. Molecular cloning of a putative receptor protein kinase gene encoded at the self-incompatibility locus of Brassica oleracea. Proc. Natl. Acad. Sci. USA. 1991, 88, 8816-8820. [CrossRef]

4. Schopfer, C.R.; Nasrallah, M.E.; Nasrallah, J.B. The Male Determinant of Self-Incompatibility in Brassica. Science 1999, 286, 1697-1700. [CrossRef] [PubMed]

5. Suzuki, G.; Kai, N.; Hirose, T.; Fukui, K.; Nishio, T.; Takayama, S.; Isogai, A.; Watanabe, M.; Hinata, K. Genomic organization of the $\mathrm{S}$ locus: Identification and characterization of genes in SLG/SRK region of $\mathrm{S}$ (9) haplotype of Brassica campestris (syn. rapa). Genetics 1999, 153, 391-403. [PubMed]

6. Kachroo, A.; Schopfer, C.R.; Nasrallah, M.E.; Nasrallah, J.B. Allele-specific receptor-ligand interactions in Brassica self-incompatibility. Science 2001, 293, 1824-1826. [CrossRef] [PubMed] 
7. Takayama, S.; Shimosato, H.; Shiba, H.; Funato, M.; Che, F.-S.; Watanabe, M.; Iwano, M.; Isogai, A. Direct ligand-receptor complex interaction controls Brassica self-incompatibility. Nature 2001, 413, 534-538. [CrossRef]

8. Kachroo, A.; Nasrallah, M.E.; Nasrallah, J.B. Self-Incompatibility in the Brassicaceae. Plant Cell 2002, 14 (Suppl. 1), S227-S238. [CrossRef]

9. Haffani, Y.; Gaude, T.; Cock, J.; Goring, D. Antisense suppression of thioredoxin h mRNA in Brassica napus cv Westar pistils causes a low level constitutive pollen rejection response. Plant Mol. Biol. 2004, 55, 619-630. [CrossRef]

10. Kakita, M.; Murase, K.; Iwano, M.; Matsumoto, T.; Watanabe, M.; Shiba, H.; Isogai, A.; Takayama, S. Two Distinct Forms of M-Locus Protein Kinase Localize to the Plasma Membrane and Interact Directly with S-Locus Receptor Kinase to Transduce Self-Incompatibility Signaling in Brassica rapa. Plant Cell 2007, 19, 3961-3973. [CrossRef]

11. Stone, S.L.; Anderson, E.M.; Mullen, R.T.; Goring, D.R. ARC1 Is an E3 Ubiquitin Ligase and Promotes the Ubiquitination of Proteins during the Rejection of Self-Incompatible Brassica Pollen. Plant Cell 2003, 15, 885-898. [CrossRef]

12. Samuel, M.A.; Chong, Y.T.; Haasen, K.E.; Aldea-Brydges, M.G.; Stone, S.L.; Goring, D.R. Cellular pathways regulating responses to compatible and self-incompatible pollen in Brassica and Arabidopsis stigmas intersect at Exo70A1, a putative component of the exocyst complex. Plant Cell 2009, 21, 2655-2671. [CrossRef] [PubMed]

13. Doucet, J.; Lee, H.K.; Goring, D.R. Pollen Acceptance or Rejection: A Tale of Two Pathways. Trends Plant Sci. 2016, 21, 53-62. [CrossRef] [PubMed]

14. Sankaranarayanan, S.; Jamshed, M.; Samuel, M.A. Degradation of glyoxalase I in Brassica napus stigma leads to self-incompatibility response. Nat. Plants 2015, 1, 15-21. [CrossRef] [PubMed]

15. Thornalley, P.J. The glyoxalase system: New developments towards functional characterization of a metabolic pathway fundamental to biological life. Biochem. J. 1990, 269, 1-11. [CrossRef] [PubMed]

16. Nasrallah, J.B.; Nasrallah, M.E. Robust Self-Incompatibility in the Absence of a Functional ARC1 Gene in Arabidopsis thaliana. Plant Cell 2014, 26, 3838-3841. [CrossRef] [PubMed]

17. Goring, D.R.; Indriolo, E.; Samuel, M.A. The ARC1 E3 Ligase Promotes a Strong and Stable Self-Incompatibility Response in Arabidopsis Species: Response to the Nasrallah and Nasrallah Commentary. Plant Cell 2014, 26, 3842-3846. [CrossRef]

18. Yamamoto, M.; Nasrallah, J.B. In planta assessment of the role of thioredoxin $\mathrm{h}$ proteins in the regulation of S-locus receptor kinase signaling in transgenic Arabidopsis thaliana. Plant Physiol. 2013, 163, 1387-1395. [CrossRef] [PubMed]

19. Murase, K.; Shiba, H.; Iwano, M.; Che, F.-S.; Watanabe, M.; Isogai, A.; Takayama, S. A membrane-anchored protein kinase involved in Brassica self-incompatibility signaling. Science 2004, 303, 1516-1519. [CrossRef]

20. Kitashiba, H.; Liu, P.; Nishio, T.; Nasrallah, J.B.; Nasrallah, M.E. Functional test of Brassica self-incompatibility modifiers in Arabidopsis thaliana. Proc. Natl. Acad. Sci. USA 2011, 108, 18173-18178. [CrossRef]

21. Chalhoub, B.; Denoeud, F.; Liu, S.; Parkin, I.A.P.; Tang, H.; Wang, X.; Chiquet, J.; Belcram, H.; Tong, C.; Samans, B.; et al. Early allopolyploid evolution in the post-Neolithic Brassica napus oilseed genome. Science 2014, 345, 950-953. [CrossRef]

22. Gao, Q.; Shi, S.; Liu, Y.; Pu, Q.; Liu, X.; Zhang, Y.; Zhu, L. Identification of a novel MLPK homologous gene MLPKn1 and its expression analysis in Brassica oleracea. Plant Reprod. 2016, 29, 239-250. [CrossRef]

23. Hanks, S.K.; Quinn, A.M. Protein kinase catalytic domain sequence database: Identification of conserved features of primary structure and classification of family members. Methods Enzymol. 1991, 200, 38-62.

24. Boisson, B.; Giglione, C.; Meinnel, T. Unexpected protein families including cell defense components feature in the N-myristoylome of a higher eukaryote. J. Biol. Chem. 2003, 278, 43418-43429. [CrossRef]

25. Safavian, D.; Zayed, Y.; Indriolo, E.; Chapman, L.; Ahmed, A.; Goring, D. RNA silencing of exocyst genes in the stigma impairs the acceptance of compatible pollen in Arabidopsis. Plant Physiol. 2015, 169, 2526-2538. [CrossRef]

26. Bower, M.S.; Matias, D.D.; Fernandes-Carvalho, E.; Mazzurco, M.; Gu, T.; Rothstein, S.J.; Goring, D.R. Two members of the thioredoxin-h family interact with the kinase domain of a Brassica $\mathrm{S}$ locus receptor kinase. Plant Cell 1996, 8, 1641-1650. 
27. Haasen, K.E.; Goring, D.R. The recognition and rejection of self-incompatible pollen in the Brassicaceae. Bot. Stud. 2010, 51, 1-6.

28. Ivanov, R.; Fobis-Loisy, I.; Gaude, T. When no means no: Guide to Brassicaceae self-incompatibility. Trends Plant Sci. 2010, 15, 387-394. [CrossRef]

29. Tantikanjana, T.; Nasrallah, M.E.; Nasrallah, J.B. Complex networks of self-incompatibility signaling in the Brassicaceae. Curr. Opin. Plant Biol. 2010, 13, 520-526. [CrossRef]

30. Li, Y.; Dai, C.; Hu, C.; Liu, Z.; Kang, C. Global identification of alternative splicing via comparative analysis of SMRT- and Illumina-based RNA-seq in strawberry. Plant J. 2017, 90, 164. [CrossRef]

31. Zavolan, M.; Van, N.E.; Gaasterland, T. Splice variation in mouse full-length cDNAs identified by mapping to the mouse genome. Genome Res. 2002, 12, 1377-1385. [CrossRef]

32. Koch, M.A.; Haubold, B.; Mitchell-Olds, T. Comparative evolutionary analysis of chalcone synthase and alcohol dehydrogenase loci in Arabidopsis, Arabis, and related genera (Brassicaceae). Mol. Biol. Evol. 2000, 17, 1483-1498. [CrossRef]

33. Nasrallah, J.B. Self-incompatibility in the Brassicaceae: Regulation and mechanism of self-recognition. Curr. Top. Dev. Biol. 2019, 131, 435-452. [CrossRef]

34. Silva, N.F.; Stone, S.L.; Christie, L.N.; Sulaman, W.; Nazarian, K.A.; Burnett, L.A.; Arnoldo, M.A.; Rothstein, S.J.; Goring, D.R. Expression of the S receptor kinase in self-compatible Brassica napus cv. Westar leads to the allele-specific rejection of self-incompatible Brassica napus pollen. Mol. Genet. Genom. MGG 2001, 265, 552-559.

35. Goring, D.R.; Glavin, T.L.; Schafer, U.; Rothstein, S.J. An S receptor kinase gene in self-compatible Brassica napus has a 1-bp deletion. Plant Cell 1993, 5, 531-539. [CrossRef]

36. Gao, C.; Ma, C.; Zhang, X.; Li, F.; Zhang, J.; Zhai, W.; Wang, Y.; Tu, J.; Shen, J.; Fu, T. The genetic characterization of self-incompatibility in a Brassica napus line with promising breeding potential. Mol. Breed. 2013, 31, 485-493. [CrossRef]

37. Sambrock, J.; Russel, D. Molecular Cloning: A Laboratory Manual, 3rd ed.; Cold Spring Harbor Laboratory Press: New York, NY, USA, 2001; Volume 3, pp. 639-646.

38. Xing, H.L.; Dong, L.; Wang, Z.P.; Zhang, H.Y.; Han, C.Y.; Liu, B.; Wang, X.C.; Chen, Q.J. A CRISPR/Cas9 toolkit for multiplex genome editing in plants. BMC Plant Biol. 2014, 14, 327. [CrossRef]

39. Gao, X.; Yan, P.; Shen, W.; Li, X.; Zhou, P.; Li, Y. Modular construction of plasmids by parallel assembly of linear vector components. Anal. Biochem. 2013, 437, 172-177. [CrossRef]

40. Franklin, T.M.; Oldknow, J.; Trick, M. SLR1 function is dispensable for both self-incompatible rejection and self-compatible pollination processes in Brassica. Sex. Plant Reprod. 1996, 9, 203-208. [CrossRef]

41. Yang, H.; Wu, J.J.; Tang, T.; Liu, K.D.; Dai, C. CRISPR/Cas9-mediated genome editing efficiently creates specific mutations at multiple loci using one sgRNA in Brassica napus. Sci. Rep. 2017, 7, 7489. [CrossRef]

42. Yang, Y.; Liu, Z.; Zhang, T.; Zhou, G.; Duan, Z.; Li, B.; Dou, S.; Liang, X.; Tu, J.; Shen, J.; et al. Mechanism of Salt-Induced Self-Compatibility Dissected by Comparative Proteomic Analysis in Brassica napus L. Int. J. Mol. Sci. 2018, 19, 1652. [CrossRef]

(C) 2019 by the authors. Licensee MDPI, Basel, Switzerland. This article is an open access article distributed under the terms and conditions of the Creative Commons Attribution (CC BY) license (http://creativecommons.org/licenses/by/4.0/). 